\title{
ON THE DOMAIN OF ABSOLUTE CONVERGENCE OF DIRICHLET SERIES IN SEVERAL VARIABLES
}

\section{GIDEON PEYSER}

Ritt [4] and Hille [3] showed that the domain of absolute convergence of a Dirichlet series in one complex variable $z=x+i y$

$$
\sum_{m=1}^{\infty} A_{m} \exp \left(-\sigma_{m} z\right)
$$

with complex $A_{m}$ and $\sigma_{m}$, is a convex domain. Hille showed further that conversely, given a convex domain in the space $E_{2}=E_{2}(x, y)$ then there exists a Dirichlet series with this domain as its domain of absolute convergence.

We shall treat here the Dirichlet series in $n$ complex variables $z_{k}=x_{k}+i y_{k}, k=1, \cdots, n$,

$$
\sum_{m=1}^{\infty} A_{m} \exp \left(-\sum_{k=1}^{n} \sigma_{m k} z_{k}\right)
$$

with complex $A_{m}$ and $\sigma_{m k}$. In $\S 1$ we show that the domain of absolute convergence of this series is convex. Conversely, given a convex domain in $E_{2 n}=E_{2 n}\left(x_{1}, y_{1}, \cdots, x_{n}, y_{n}\right)$ and a preassigned sequence of exponents $\left\{\sigma_{m k}\right\}$ we construct a Dirichlet series of the given type with the given domain as its domain of absolute convergence, provided the sequence $\left\{\sigma_{m k}\right\}$ satisfies a certain set of conditions. In $\$ 2$ we construct a maximal domain of ordinary convergence. For one complex variable this reduces to a simplified construction of the maximal domain of convergence as given by Hille. We also find a relationship between the domain of absolute convergence and the maximal domain of convergence. For one complex variable this relationship reduces to a refinement of a result by Gallie [2] and for one variable with positive increasing exponents it reduces to the classical theorem regarding the relationship between the abscissas of absolute and ordinary convergence.

1. ThEOREM 1. The domain of absolute convergence of series (1) is convex.

Proof. Let $\left(z_{11}, \cdots, z_{n 1}\right)$ and $\left(z_{12}, \cdots, z_{n 2}\right)$ be any two points inside the domain of absolute convergence of series (1). Let $\left(w_{1}, \cdots, w_{n}\right)$ be any point on the straight line connecting these two points. Then

Received by the editors May 29, 1957 and, in revised form, September 23, 1957. 


$$
\begin{array}{ll}
w_{1}=t z_{11}+(1-t) z_{12} & \\
\cdot & 0 \leqq t \leqq 1 . \\
w_{n}=t z_{n 1}+(1-t) z_{n 2} &
\end{array}
$$

Therefore

$$
\begin{aligned}
& \sum_{m=1}^{\infty}\left|A_{m} \exp \left(-\sum_{k=1}^{n} \sigma_{m k} w_{k}\right)\right| \\
& \quad=\sum_{m=1}^{\infty}\left|A_{m} \exp \left(-\sum_{k=1}^{n} \sigma_{m k} z_{k 1}\right)\right|^{t}\left|A_{m} \exp \left(-\sum_{k=1}^{n} \sigma_{m k} z_{k 2}\right)\right|^{1-t} \\
& \leqq \sum_{m=1}^{\infty}\left|A_{m} \exp \left(-\sum_{k=1}^{n} \sigma_{m k} z_{k 1}\right)\right|+\sum_{m=1}^{\infty}\left|A_{m} \exp \left(-\sum_{k=1}^{n} \sigma_{m k} z_{k 2}\right)\right|
\end{aligned}
$$

Inequality (2) is based on the simple estimate that for $a \geqq 0, b \geqq 0$ and $0 \leqq t \leqq 1$ we have $a^{t} b^{1-t} \leqq \max (a, b) \leqq a+b .{ }^{1}$ Therefore series (1) converges absolutely at $\left(w_{1}, \cdots, w_{n}\right)$ which proves the theorem.

For the converse part we introduce a sequence of real numbers $\Gamma=\left\{\lambda_{m}\right\}$ with the following properties.

(3a) If $\lambda \in \Gamma$ then also $r \lambda \in \Gamma$ where $r$ is any positive or negative integer.

(3b) $\sum_{m=1}^{\infty} \exp \left(-\left|\lambda_{m}\right|\right)$ has a finite exponent of convergence $\rho$ i.e. $\sum_{m=1}^{\infty} \exp \left[-\left|\lambda_{m}\right|(\rho+\epsilon)\right]$ converges for all $\epsilon>0$ and diverges for all $\epsilon<0 .^{2}$

As examples of such sequences we have $\{ \pm \log m\}$ where $m$ are the positive integers, and $\left\{ \pm \log p^{m}\right\}$ where $p^{m}$ are the positive integral powers of all the primes. The corresponding exponent of convergence in both these examples is $\rho=1$.

TheOREM 2. Given a convex domain $D$ in $E_{2 n}$ and given a sequence of real numbers $\Gamma=\left\{\lambda_{m}\right\}$ having the properties (3) then there exists a Dirichlet series of the form (1) with $\operatorname{Re} \sigma_{m k} \in \Gamma$ and $\operatorname{Im} \sigma_{m k} \in \Gamma$ such that $D$ is its domain of absolute convergence.

Proof. Without loss of generality we assume that the origin of $E_{2 n}$ is an interior point of $D$.

First we construct the required series for the case that the boundary of $D$ is a plane which we denote by $A$

$$
a_{1} x_{1}+b_{1} y_{1}+\cdots+a_{n} x_{n}+b_{n} y_{n}+c=0 \text {. }
$$

We may assume that $c>0$.

1 This inequality was already used by Hille [3] and Ritt [4] for the case of one complex variable.

${ }_{2} \rho$ is actually the abscissa of convergence of $\sum_{m-1}^{\infty} \exp \left(-\left|\lambda_{m}\right| x\right)$. 
Now construct a sequence of approximating "rational planes" $A_{m}$

$$
a_{1 m} x_{1}+b_{1 m} y_{1}+\cdots+a_{n m} x_{n}+b_{n m} y_{n}+c_{m}=0
$$

with $c_{m}>0$, and such that $a_{i m}$ and $b_{i m}$ are integers and the planes $A_{m}$ tend towards the plane $A$ for $m \rightarrow \infty$. The series

$$
\begin{aligned}
\sum_{m=1}^{\infty} t_{m}=\sum_{m=1}^{\infty} \exp \left\{-\left|\lambda_{m}\right|\left[\left(a_{1 m}-i b_{1 m}\right) z_{1}\right.\right. & +\cdots \\
& \left.\left.+\left(a_{n m}-i b_{n m}\right) z_{n}+c_{m}+\rho\right]\right\}
\end{aligned}
$$

solves our problem for the domain with the plane $A$ as its boundary. In fact

$$
\sum_{m=1}^{\infty}\left|t_{m}\right|=\sum_{m=1}^{\infty} \exp \left[-\left|\lambda_{m}\right|\left(a_{1 m} x_{1}+\cdots+b_{n m} y_{n}+c_{m}+\rho\right)\right] .
$$

If $\left(x_{1}, \cdots, y_{n}\right)$ is a point on that side of plane $A$ which does not include the origin, then series (5), except for a finite number of terms, is termwise larger than the terms of $\sum_{m=1}^{\infty} \exp \left[-\left|\lambda_{m}\right|(\rho-\eta / 2)\right]$ where $\eta$ is the distance between the given point and the plane $A$.

If the point lies on the side of $A$ which includes the origin, then series (5), except for a finite number of terms, is termwise less than the terms of $\sum_{m=1}^{\infty} \exp \left[-\left|\lambda_{m}\right|(\rho+\eta / 2)\right]$. This concludes the case of the boundary of $D$ being a plane.

Now, if $D$ is any convex domain with boundary $B$ then we select a sequence of points $\left\{\left(\bar{x}_{1 r}, \cdots, \bar{y}_{n r}\right)\right\}$ dense on the boundary $B$ and a sequence of points $\left\{\left(x_{1 r}, \cdots, y_{n r}\right)\right\}$ dense in $D$.

Through each point of the set dense on $B$ we construct a plane of support to the domain $D$. Denote these planes by $A^{r}$. For each such plane of support we construct a sequence of approximating rational planes of the form (4) which we denote $A_{m}^{r}$

$$
a_{a_{m}}^{r} x_{1}+\cdots+b_{n m}^{r} y_{n}+c_{m}^{r}=0 .
$$

We impose on these approximating planes the additional restrictions that for all $k$, the $k$ points $\left(x_{11}, \cdots, y_{n 1}\right), \cdots,\left(x_{1 k}, \cdots, y_{n k}\right)$ of the selected dense set in $D$ lie on that side of the planes $A_{k}^{r}, r=1,2, \ldots$ which includes the origin.

The series

$$
\sum_{m=1}^{\infty} t_{m}=\sum_{m=1}^{\infty} \sum_{r=1}^{m} 2^{-r} \exp \left\{-\left|\lambda_{m}\right|\left[\left(a_{1 m}^{r}-i b_{1 m}^{r}\right) z_{1}+\cdots\right.\right.
$$

$$
\left.\left.+\left(a_{n m}^{r}-i b_{n m}^{r}\right) z_{n}+c_{m}^{r}+\rho\right]\right\}
$$


solves our problem. In fact, if a point $\left(x_{1}, \cdots, y_{n}\right)$ lies outside $D$ then it lies on that side of one of the constructed planes of support that does not include the origin, at a distance $\eta>0$ from that plane. There exists therefore a subseries of (6) the terms of which at this point are absolutely larger than the terms of

$$
2^{-r} \sum_{m=r}^{\infty} \exp \left[-\left|\lambda_{m}\right|(\rho-\eta / 2)\right]
$$

starting from a certain term.

If the given point is interior to $D$ then it lies inside a simplex whose $2 n+1$ vertices belong to the selected dense set in $D$. The point is therefore at a distance larger than $\eta>0$ from all the planes of all the approximating sequences, except possibly at most from a fixed number of planes at the beginning of each approximating sequence. $\eta$ can be taken as the minimum distance between the point and the sides of the simplex. The absolute values of the terms of (6) at the given point, except for a finite number, add up to less than

$$
\sum_{m=1}^{\infty} \exp \left[-\left|\lambda_{m}\right|(\rho+\eta)\right]
$$

This completes the proof.

Clearly none of the above constructions are unique.

2. We construct now a maximal domain of ordinary convergence for a given series of form (1) i.e. a domain outside which the series diverges. For every term of (1) we construct a corresponding halfspace:

$$
\log \left|A_{m} \exp \left(-\sum_{k=1}^{n} \sigma_{m k} z_{k}\right)\right|
$$

$$
\equiv \log \left|A_{m}\right|-\sum_{k=1}^{n}\left(\alpha_{m k} x_{k}-\beta_{m k} y_{k}\right) \leqq 0
$$

where $\sigma_{m k}=\alpha_{m k}+i \beta_{m k}$.

This sequence of halfspaces possesses limiting halfspaces, which may also be the whole space and the empty set. Let $M$ be the intersection of all limiting halfspaces. ${ }^{3}$ Clearly $M$ is closed and convex.

THEOREM 3. The series (1) diverges at all points outside of $M$.

Proof. If $\left(x_{1}, \cdots, y_{n}\right)$ lies outside of $M$ then it lies outside an

${ }^{3}$ For the case of two complex variables and positive increasing exponents see Artémiadès [5]. 
infinite number of the halfspaces (7). Therefore an infinite number of the terms of the series are absolutely larger than 1 , which completes the proof.

Further we have the following relationship between the domain of absolute convergence and the maximal domain of convergence $M$.

THEOREM 4. If $\rho$ is the exponent of convergence of

$$
\sum_{m=1}^{\infty} \exp \left[-\left(\left|\sigma_{m 1}\right|^{2}+\cdots+\left|\sigma_{m n}\right|^{2}\right)^{1 / 2}\right]
$$

then (1) converges absolutely at any point of $M$ whose distance from the boundary is larger than $\rho$.

Proof. Any point of $M$ whose distance from the boundary is $\rho+2 \epsilon$ with $\epsilon>0$, is at a distance of at least $\rho+\epsilon$ from all but a finite number of the planes that are the boundaries of the halfspaces (7). Therefore, except for a finite number of terms

$$
\left(\sum_{k=1}^{n}\left|\sigma_{m k}\right|^{2}\right)^{-1 / 2}\left[\log \left|A_{m}\right|-\sum_{k=1}^{n}\left(\alpha_{m k} x_{k}-\beta_{m k} y_{k}\right)\right] \leqq-(\rho+\epsilon) .
$$

Therefore, except for a finite number of terms

$$
\begin{aligned}
\left|A_{m}\right| \exp \left[-\sum_{k=1}^{n}\left(\alpha_{m k} x_{k}-\beta_{m k} y_{k}\right)\right] & \\
& \leqq \exp \left[-\left(\sum_{k=1}^{n}\left|\sigma_{m k}\right|^{2}\right)^{1 / 2}(\rho+\epsilon)\right] .
\end{aligned}
$$

This proves the theorem.

Remarks. (1) In the case of one complex variable, Gallie [1] proved that the series converges absolutely at any point of $M$ whose distance from the boundary is larger than $\lim \sup _{m \rightarrow \infty}(\log m) /\left|\sigma_{m}\right|$. However it is readily verified that $\rho \leqq \lim \sup _{m \rightarrow \infty}(\log m) /\left|\sigma_{m}\right|$. One can easily construct an example such that actually

$$
\rho<\limsup _{m \rightarrow \infty}(\log m) /\left|\sigma_{m}\right| \text {. }
$$

(2) If $\left(\sum_{k=1}^{n}\left|\sigma_{m k}\right|^{2}\right)^{1 / 2}$ is a sequence of numbers increasing steadily to infinity, then it follows from a classical theorem, see Hardy-Riesz $\left[2\right.$, p. 8], that $\rho=\lim \sup _{m \rightarrow \infty}(\log m) /\left(\sum_{k=1}^{n}\left|\sigma_{m k}\right|^{2}\right)^{1 / 2}$. For one complex variable with positive exponents increasing steadily to infinity, Theorem 4 reduces therefore, to the classical theorem regarding the relationship between the abscissas of absolute and ordinary convergence, see Hardy-Riesz [2, p. 9].

(3) If $\rho=0$ then the maximal domain of convergence, the domain 
of ordinary convergence and the domain of absolute convergence coincide.

\section{REFERENCES}

1. T. M. Gallie, Region of convergence of Dirichlet series with complex exponents, Proc. Amer. Math. Soc. vol. 7 (1956) pp. 627-629.

2. G. H. Hardy and M. Riesz, The general theory of Dirichlet's series, Cambridge Tracts in Math. and Math. Physics, 1952.

3. E. Hille, Note on Dirichlet's series with complex exponents, Ann. of Math. vol. 25 (1924) pp. 261-278.

4. J. F. Ritt, On a class of linear homogeneous differential equations of finite order with constant coefficients, Trans. Amer. Math. Soc. vol. 18 (1917) pp. 27-49.

5. N. Artémiadès, Sur les séries de Dirichlet d̀ deux variables, Bull. Sci. Math. (2) vol. 77 (1953) pp. 48-51.

Pratt Institute 